\title{
Binary pulsars before spin-up and PSR 1820 - 11
}

\author{
E. S. Phinney ${ }^{1}$ and F. Verbunt ${ }^{1,2}$ \\ ${ }^{1}$ Theoretical Astrophysics, 130-33, California Institute of Technology, Pasadena CA 91125, USA \\ ${ }^{2}$ Astronomical Institute, Postbox 80.000, NL-3508 TA Utrecht, The Netherlands
}

Accepted 1990 November 21. Received 1990 November 15; in original form 1990 October 22

\begin{abstract}
SUMMARY
We argue that PSR 1820-11 may be a young pulsar, with a main-sequence companion, and that the system is the progenitor of a low-mass X-ray binary, rather than the descendant of one. If it is not, such a system should soon be found. The small number of antediluvian binary pulsars suggests that most, but not all neutron stars have some torque decay after birth.
\end{abstract}

\section{INTRODUCTION}

Radio pulsars in binaries are thought to have descended from X-ray binaries. The mass of the donor in the X-ray binary determines the properties of the radio pulsar binary which ensues. Table 1 lists the binary radio pulsars in the galactic disc relevant to our discussion, together with their properties. An X-ray binary with a massive donor may evolve into binary with a (mildly) spun-up radio pulsar and either a massive white-dwarf companion in a circular orbit, like PSR $0655+64$, or a neutron star companion in a very eccentric orbit, like PSR $1913+16$, PSR $2303+46$ and PSR $1534+12$. An $X$-ray binary with a low-mass giant donor will evolve into a binary containing a spun-up neutron star and a white-dwarf companion, of $0.2-0.4 M_{\odot}$, in a wide circular orbit, like PSR $1855+09$, PSR 1953+29, PSR $1831-00$ and PSR $0820+02$. For a review of the evolutionary scenarios for radio pulsars in binaries, see Van den

Table 1. Properties of some radio binary pulsars.

$\begin{array}{lrrrrllll}\text { pulsar } & P & \log P / \dot{P} & \log B & P_{b} & e & l(M) & M_{c} & \text { ref. } \\ & \mathrm{ms} & \mathrm{s} & \mathrm{G} & \mathrm{d} & & M_{\odot} & M_{\odot} & \\ 1855+09 & 5.4 & 17.4 & 8.5 & 12.33 & 0.000021 & 0.0056 & 0.22 & 1 \\ 1953+29 & 6.1 & 17.3 & 8.6 & 117.35 & 0.00033 & 0.0024 & 0.21^{*} & 2 \\ 1831-00 & 521.0 & 16.6 & 10.9 & 1.81 & <0.004 & 0.00012 & 0.07^{*} & 3 \\ 0820+02 & 864.9 & 15.9 & 11.5 & 1232.34 & 0.0119 & 0.0030 & 0.23^{*} & 4 \\ & & & & & & & & \\ 1534+12 & 37.9 & & & 0.42 & 0.27 & 0.32 & 1.7^{*} & 5 \\ 1913+16 & 59.0 & 15.9 & 10.3 & 0.32 & 0.6171 & 0.1322 & 1.39 & 6 \\ 0655+64 & 195.6 & 17.5 & 10.0 & 1.03 & 0.0000075 & 0.0712 & 0.8 & 7 \\ 2303+46 & 1066.4 & 15.4 & 11.8 & 12.34 & 0.6584 & 0.2463 & 1.5 & 8 \\ 1820-11 & 279.8 & 14.3 & 11.8 & 357.76 & 0.79462 & 0.068 & 0.8^{*} & 9\end{array}$

Companion masses $M_{\mathrm{c}}$ marked * were estimated from the mass function $f(M)$ for a $1.4 M_{\odot}$ pulsar mass, and an inclination of $60^{\circ}$. References: 'Segelstein et al. (1986), Ryba \& Taylor (1991); ${ }^{2}$ Boriakoff, Bucchesi \& Fauci (1983), Rawley, Taylor \& Davis (1986); ${ }^{3}$ Dewey et al. (1986), Taylor \& Dewey (1988); ${ }^{4}$ Manchester et al. (1980, 1983); ${ }^{5}$ Wolszczan (1990); 'Hulse \& Taylor (1975), Taylor \& Weisberg (1989); ${ }^{7}$ Damashek et al. (1982), Jones \& Lyne (1988); ${ }^{8}$ Stokes, Taylor \& Dewey (1985), Lyne \& Bailes (1990); ${ }^{9}$ Lyne \& McKenna (1989).
Heuvel (1987) and Verbunt (1990). In Section 2 we argue that one would expect $\sim 1$ binary pulsar to have been discovered before its X-ray luminous mass-transfer phase. We show that PSR 1820-11 could well be such an antediluvian pulsar. A discussion of tests and the consequences for neutron star field decay is given in Section 3 .

\section{BEFORE THE DELUGE: PSR 1820 - 11}

A possible scenario for the formation of low-mass X-ray binaries (LMXBs) with giant donors is as follows (see Verbunt 1990, section 5.1): a rapidly expanding massive supergiant engulfs a low-mass companion. This companion spirals in towards the core of the supergiant, and forms a close binary with it. The core then explodes as a supernova, leaving a neutron star in a very eccentric orbit around the low-mass main-sequence star. The binary becomes an X-ray binary only after the main-sequence star evolves into a giant, $\sim 10^{10} \mathrm{yr}$ later.

In previous discussions of this scenario, it appears to have been overlooked that immediately after the supernova explosion, and long before mass transfer begins, the neutron star in its eccentric orbit around the main-sequence star, would be observable as a radio pulsar until it reached the death-line. For a constant magnetic field of about $10^{12} \mathrm{G}$, this would taken about $10^{8} \mathrm{yr}$.

How many such systems do we expect to observe? The answer depends on the fraction of low-mass X-ray binaries and - via them - binary radio pulsars formed via this scenario. Let us assume for the moment that this fraction is substantial (we discuss this assumption in Section 3). The galactic birthrate of single pulsars whose radio beam crosses the Earth is of order $R_{\mathrm{p}}=3 \times 10^{-3} \mathrm{yr}^{-1}$ (Narayan \& Ostriker 1990; when allowance is made for pulsars whose beam does not cross the Earth, the total pulsar birthrate is comparable to the supernova rate). The birthrate of observable binary pulsars with a low-mass white-dwarf companion is $R_{\mathrm{b}} \gtrsim 3 \times 10^{-5} \mathrm{yr}^{-1} \quad$ (Kulkarni \& Narayan 1988). With $N_{\mathrm{p}} \simeq 500$ young radio pulsars now known, we therefore 
expect a detection of $\left(R_{\mathrm{b}} / R_{\mathrm{p}}\right) N_{\mathrm{p}} \simeq \mathrm{a}$ few young radio pulsars with a low-mass main-sequence companion. This argument is independent of the beaming fractions of the various types of pulsars, but does depend on the uncertain birthrate estimates for binary pulsars (uncertain because of smallnumber statistics).

Such a system may already have been discovered: PSR 1820-11. We note that PSR 1820-11 is the only Galactic binary pulsar which has a period shorter than allowed by the conventional 'rebirth line', clear evidence that it is not recycled. The magnetic field and the rotation period of this pulsar are like those of young isolated radio pulsars. Since the orbit is highly eccentric, its companion cannot be a white dwarf formed after the pulsar, for the orbit would then have circularized during the red giant phase (either by spiralin within a massive star, leading to a massive white dwarf as for PSR $0655+64$, or by tidal circularization during transfer in a low-mass binary, as for the first four pulsars in Table 1). In addition, the mass function is less than those of the pulsars with neutron star companions (PSR 1913+16, PSR $2303+46$ ), but is easily compatible with a $\sim 1 M_{\odot}$ main-sequence star. The galactic latitude of the binary is 0.95 , which at a distance of $11 \mathrm{kpc}$ corresponds to a distance to the galactic plane $z \simeq 180 \mathrm{pc}$, again quite compatible with the scaleheight of young isolated pulsars.

If the pulsar formed without a velocity kick, then adopting a pulsar mass of $1.4 M_{\odot}$, and a main-sequence star companion of $M_{\mathrm{c}}=1 M_{\odot}$, the present orbital period and eccentricity require the mass of the pre-supernova core to be $3.3 M_{\odot}$, and the pre-supernova orbital period about $25 \mathrm{~d}$ (see equation 4 of Dewey \& Cordes 1987). The core could have had a somewhat higher mass, if the neutron star received a kick at birth which helped to keep the binary intact ( $c f$. Bailes 1989). These values seem not unreasonable. It appears quite possible then that PSR $1820-11$ is the first young radio pulsar to be discovered in a binary before its companion transfermed mass to it.

\section{DISCUSSION}

What does the future hold for PSR 1820-11? If its magnetic field does not decay, the pulsar will cross the death line in $10^{8} \mathrm{yr}$. It will then cease to exist as a radio pulsar for $\sim 10^{10} \mathrm{yr}$ until its companion evolves into a red giant. The ensuing accretion will then spin it back up to an equilibrium period 1 s (Pringle \& Rees 1972; Davidson \& Ostriker 1973). After accretion ceased, the resulting system would be strikingly similar to PSR $0820+02$.

This similarity suggests to us that PSR $0820+02$ is in fact a recycled $\sim 10^{10}$ yr old neutron star whose $3 \times 10^{11} \mathrm{G}$ magnetic field has not decayed. If, on the other hand, the magnetic field of PSR 1820-11 does decay to $\sim 10^{10}-10^{8} \mathrm{G}$, it could be a progenitor only of the low-field binary pulsars listed in Table 1 . In a variant of the scenario that we assumed in Section 2, the supergiant evolves into a massive white dwarf at first, and is only transformed into a neutron star during the phase of mass transfer, via accretioninduced collapse. This variant was popular when it was thought that old neutron stars could not have a strong enough magnetic field to be radio pulsar (Van den Heuvel 1987). Indeed, if all neutron star magnetic fields decayed on a time-scale of $10^{7} \mathrm{yr}$, this would be the only way to form systems like PSR $0820+02$. The motivation for accretioninduced collapse has recently declined, however, as it has been shown that old neutron stars can have fields as high as $10^{10} \mathrm{G}$ (Kulkarni 1986) and even $10^{12} \mathrm{G}$ (Verbunt, Wijers \& Burm 1990). We suggest therefore, that all, or many, of even the high-field radio pulsars in binaries were formed as neutron stars directly in the collapse of massive stars.

Strong support for this possibility would be obtained if it were proven that the companion to PSR $1820-11$ is a mainsequence star. The other possibility is that PSR $1820-11$ is similar to PSR $1913+16$, and has a neutron star companion. How can one discriminate between a neutron star and a main-sequence star companion to the radio pulsar? Direct identification of radiation from the main-sequence star seems unlikely, due to the crowded field and high interstellar extinction to be expected (Lyne \& McKenna 1989). The best possibility is the measurement of the periastron advance. Unless the companion is highly evolved, the classical rate of advance of apsides, $\sim 10^{-8} \mathrm{deg} \mathrm{yr}^{-1}$ will be negligible compared to the relastivistic rate of advance, $\sim 3 \times 10^{-4} \mathrm{deg}$ $\mathrm{yr}^{-1}$. The latter will give an estimate of the total mass of the binary, and hence the mass of the companion of the radio pulsar, assuming the latter to be about $1.4 M_{\odot}$. A companion mass distinctly lower than $1.4 M_{\odot}$ would strongly suggest a main-sequence companion.

Finally, we note that if no magnetic fields decayed, roughly equal numbers of pulsars should be found in low mass binaries before mass transfer and after, since the time to reach the death line depends only on the magnetic field. Could it be true that no magnetic fields decay? Of the five low mass binary pulsars in Table 1 , only PSR 1820 - 11 can be antediluvian: all the others have circularized orbits (optical observations directly confirm that the companions of PSRs $0280+02$ and $0655+64$ and $1844+09$ are white dwarfs - Kulkarni 1986; Callanan et al. 1989). Thus at most $1 / 5$ low-mass binary pulsars are antediluvian. The admittedly meagre statistics thus suggest that most, but not all neutron star magnetic fields to decay. Alternatively, the ratio could be explained if all magnetic fields decayed by a factor of 4 over $10^{10} \mathrm{yr}$. Since the time to reach the death line $B / P^{2}=$ constant scales as $B^{-1}$, reducing the dipole moment of recycled pulsars by a factor of 4 would ensure that they lasted four times as long, and hence were four times more numerous than their antediluvian progenitors.

In high-mass binaries containing two neutron stars, the situation is complicated by three factors. (i) Antediluvian pulsars with massive companions may be hidden by absorption and dispersion in the dense wind of the companion star. (ii) The lifetime of the companion may also be less than the active lifetime of the radio pulsar. (iii) The second supernova may disrupt the binary. The ratio of postdiluvian to antediluvian massive binary pulsars is therefore unpredictable. However, it is interesting to note that of the four pulsars in high-mass binaries, there is one which might be unaffected by mass transfer, namely PSR $2303+46$. This has a magnetic field and pulse period simar to those of young single radio pulsars, though unlike PSR 1820-11, it has a period longer than that given by the standard spin-up line, so the case is less clear cut. Thus it is possible, though not required, that the neutron star companion of this pulsar is the spun-up neutron star, and the observed pulsar the younger one. 


\section{ACKNOWLEDGMENTS}

We thank Shri Kulkarni for discussions. ESP was supported in part by NSF PYI grant AST 84-51725 and the Alfred P. Sloan Foundation. FV thanks Caltech for partial support.

\section{REFERENCES}

Bailes, M., 1989. Astrophys. J., 342, 917.

Bariakoff, V., Buccheri, R. \& Fauci, F., 1983. Nature, 304, 417.

Callanan, P. J.,Charles, P. A., Hassall, B. J. M., Machin, G., Mason, K. O., Naylor, T., Smale, A. P. \& van Paradijs, J., 1989. Mon. Not. R. astr. Soc., 238, 25P.

Damashek, M., Backus, P. R., Taylor, J. H. \& Burkhardt, R. K., 1982. Astrophys. J., 253, L57.

Davidson, K. \& Ostriker, J. P., 1973. Astrophys. J., 179, 585.

Dewey, R. J. \& Cordes, J. M., 1987. Astrophys. J., 321, 780.

Dewey, R. J., Maguire, C. M., Rawley, L. A., Stokes, G. H. \& Taylor, J. H., 1986. Nature, 322, 712.

Hulse, R. A. \& Taylor, J. H., 1975. Astrophys. J., 195, L51.

Jones, A. W. \& Lyne, A. G., 1988. Mon. Not. R. astr. Soc., 232, 473.

Kulkarni, S. R., 1986. Astrophys. J., 306, L85.

Kulkarni, S. R. \& Narayan, R., 1988. Astrophys. J., 335, 755.
Lyne, A. G. \& Bailes, M., 1990. Mon. Not. R. astr. Soc., 246, 15P.

Lyne, A. G. \& McKenna, J., 1989. Nature, 340, 367.

Manchester, R. N., Newton, L. M., Cooke, D. J. \& Lyne, A. G., 1980. Astrophys. J., 236, L25.

Manchester, R. N., Newton, L. M., Cooke, D. J., Backus, P. R., Damashek, M., Taylor, J. H. \& Condon, J. J., 1983. Astrophys. J., 268, 832 .

Narayan, R. \& Ostriker, J. P., 1990. Astrophys. J., 352, 222.

Pringle, J. E. \& Rees, M. J., 1972. Astr. Astrophys., 21, 1.

Rawley, L. A., Taylor, J. H. \& Davis, M. M., 1986. Nature, 319, 383.

Ryba, M. F. \& Taylor, J. H., 1991. Astrophys. J., in press.

Segelstein, D. J., Rawley, L. A., Stinebring, D. R., Fruchter, A. S. \& Taylor, J. H., 1986. Nature, 322, 714.

Stokes, G. H., Taylor, J. H. \& Dewey, R. J., 1985. Astrophys. J., 294, L21.

Taylor, J. H. \& Dewey, R. J., 1988. Astrophys. J., 332, 770.

Taylor, J. H. \& Weisberg, J. M., 1989. Astrophys. J., 345, 434.

Van den Heuvel, E. P. J., 1987. The Origin and Evolution of Neutron Stars, IAU Symp. No. 125, p. 393, eds Helfand, D. J. \& Huang, J.-H., Reidel, Dordrecht.

Verbunt, F., 1990. Neutron Stars and their Birth Events, p. 179, ed. Kundt, W., Kluwer, Dordrecht.

Verbunt, F., Wijers, R. A. M. J. \& Burm, H. M. G., 1990. Astr. Astrophys., 234, 195.

Wolszczan, A., 1990. IAU Circ. No. 5073. 